\title{
Barandillas de protección personal con solicitación dinámica
}

\author{
Personal protection guardrails for dynamic forces \\ L. C. Pomares ${ }^{(*)}$, R. Irles(*), E. G. Segovia(*), D. Boixader(**)
}

\section{RESUMEN}

Las barandillas de seguridad con solicitación dinámica, tipos B y C según UNE-EN 13374, son el conjunto de componentes destinados a la protección colectiva de las personas contra las caídas a un nivel inferior y a retener materiales. Frecuentemente incorporan una red de seguridad.

En este artículo se analizan aspectos fundamentales como su diseño, sus requisitos mecánicos y la solución a la problemática que suponen los soportes o puntos duros capaces de producir lesiones al accidentado.

Para este fin, se ha estudiado el fenómeno de la caída de un lastre sobre tales redes con distintos modelos numéricos de elementos finitos en régimen dinámico $y$ mediante consideraciones analíticas cinemáticas, obteniendo conclusiones útiles como la forma geométrica necesaria, la escuadría de los perfiles que constituyen el bastidor de la red, y los factores de impacto que sufren los accidentados. Ello permite sugerir mejoras al texto normativo.

\section{SUMMARY}

Safety guardrails with dynamic loads, B and $C$ types conforming EN 13374, are devices used as collective protection for people against height falls and for retaining materials. This system can include a safety net anchored to a support structure for its vertical use (U system, EN 1263).

In this paper, fundamental aspects are analysed, as design, mechanical requirements and solution for the hard points that arise at supports of the system, able to seriously injure falling people.

So, a ballast fall against this system has been studied, by modelling it with dynamic finite elements and analytical kinetic considerations, to obtain useful conclusions about geometry, cross section of the supports, maximum deflection and impact factor over the injured. It allows to suggest normative text improvements.

Keywords: Guardrail; safety; net; fall; impact.

Palabras clave:
caída; impacto.

(*) Universidad de Alicante (España).

(**) Universidad Politécnica de Cartagena (España).

Publicado online/

Published online: 28 jan 2013 
1. Clasificación de las barandiIlas, según UNE-EN 13374.

\section{INTRODUCCIÓN}

Dentro de las actividades productivas humanas habituales, la industria de la construcción ha sido y sigue siendo una de las que más accidentes graves han generado. Según estadísticas del Ministerio de Trabajo en España, promediando los últimos 5 años, y debidos exclusivamente a caídas a distinto nivel, tenemos anualmente 2.042 accidentes graves y 114 mortales, que suponen un $26,7 \%$ del total de graves y un $13,5 \%$ del total de mortales, respectivamente.

Para evitar caídas de altura de personas y objetos a un nivel más bajo desde tejados, bordes de forjado, escaleras y otras áreas donde se requiera la protección, durante las últimas décadas se han venido utilizando en España y en otros países, como protección colectiva, sistemas de redes de seguridad y barandillas.

Los primeros estudios experimentales localizados sobre estos dispositivos son los de Spieker (1) y Becker (2) realizados en los años 60 y 70 sobre redes de seguridad horizontales. Los primeros de carácter teórico, igualmente sobre redes, se deben a Lefevre (3) y Paureau (4) (5) poco después. En décadas más recientes empiezan a publicarse algunos trabajos más de carácter mixto en el ámbito de la seguridad en construcción y en otros con problemas similares de retención de objetos. Entre ellos, y en relación con los sistemas de protección colectiva citados, pueden indicarse los trabajos de Saiz (6) (7), Irles (8), y Segovia (9) sobre redes; y más recientemente los de Lozano (10) y González (11) sobre barandillas con solicitación estática, varios de ellos en relación también con varios de los grupos de trabajo del subcomité SC2 del CTN 81 de AENOR.

Entre las primeras normativas reguladoras de estos dispositivos cabe citar la OGSHT (12) con muy pocas especificaciones técnicas (con la notable excepción de las cargas de diseño de las barandillas), la nota técnica de Bellmunt (13) y UNE 81650-80 (14) para ensayo de redes de seguridad. La Ley 31/1995 de Prevención de Riesgos Laborales (15) y los decretos para su desarroIlo no aportaron mayores especificaciones técnicas sobre los mismos. La legislación específica vigente, mucho más detallada, llegó poco después con UNE-EN 1263, partes 1 (16) y 2 (17), para las redes de seguridad, y con UNE-EN 13374 (18) para las barandillas.

Según esta última, existen tres tipos de barandillas $\mathrm{A}, \mathrm{B}$ y $\mathrm{C}$, en función del ángulo del plano de trabajo y altura de caída (Figura 1). Las de tipo A deben resistir adecuada- mente una carga estática de $0,3 \mathrm{kN}$, las de tipo $\mathrm{B}$ deben absorber de forma adecuada una energía cinética de 1.100 julios y las de tipo $C$, que se analizan detalladamente a continuación, deben ser capaces de absorber adecuadamente una energía cinética de 2.200 julios.

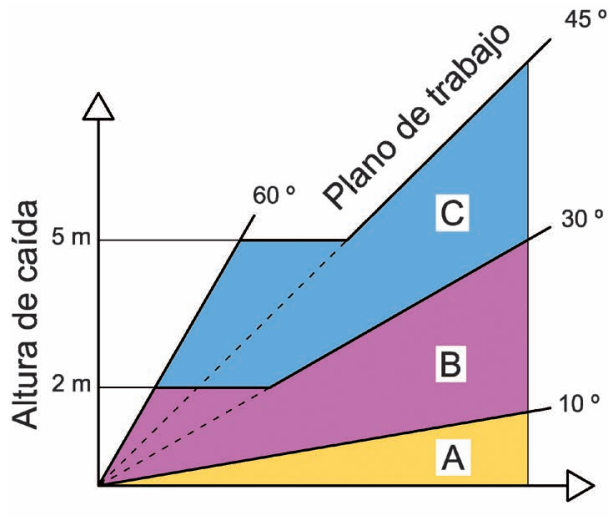

Ni en UNE-EN 13374, ni en la normativa de seguridad vigente en España, ni en los trabajos anteriores de los grupos de normalización nacionales, se define su diseño más allá de algunas especificaciones geométricas, ni se establece solución para los puntos duros que suponen los soportes de las barandillas. Limitándose sólo (18) a establecer los requisitos de comportamiento mediante la superación de un ensayo dinámico para las clases B (con saco esferocónico) y $\mathrm{C}$ con lastre cilíndrico normalizado en el que se fija en un mínimo de 200 $\mathrm{mm}$ el valor de flecha para la clase C (100 $\mathrm{mm}$ para la clase B), en cualquier parte del sistema a $200 \mathrm{~mm}$ sobre su borde inferior. Este valor será cuestionado y analizado pormenorizadamente en este artículo, en relación con un comportamiento suficientemente blando del sistema de protección, consiguiendo así que el accidentado sufra un factor de impacto suficientemente bajo, entendido éste como el cociente entre la máxima deceleración instantánea y el valor de la gravedad.

Para conseguir garantizar este requisito, en los trabajos previos a la redacción de este artículo y como consecuencia de los resultados obtenidos en los primeros estudios con modelo numérico, procedentes de la tesis doctoral del primer autor "Análisis dinámico y criterios de diseño para barandillas de protección personal sometidas a impacto", se ha manejado un diseño específico de los soportes, sujeto a patente, adecuado para dar solución a las posibles colisiones del accidentado sobre los soportes o puntos duros del sistema que pueden producirle lesiones graves o la muerte, escollo que la propia norma recoge como asunto pendiente en su versión actual. 


\section{ESTUDIOS REALIZADOS}

Los ensayos establecidos en UNE-EN 13374 para barandillas tipo $\mathrm{C}$ utilizan un lastre cilíndrico de $75 \mathrm{Kg}, 100 \mathrm{~cm}$ de longitud y 30 $\mathrm{cm}$ de diámetro, revestido con una lámina de caucho, definido en UNE-EN 1263 (parte 1) para los ensayos sobre redes de seguridad tipo U. Sin embargo, para otros tipos de redes, ésta última dispone un lastre esférico de $100 \mathrm{Kg}$ y $50 \mathrm{~cm}$ de diámetro. El valor de $75 \mathrm{Kg}$ puede quedar escaso para la simulación del peso de una persona que sufra una caída sobre este dispositivo. Por otra parte, su impacto no lateral (de cabeza u hombros, o de pies) sobre el mismo resulta más asimilable al efecto del lastre esférico que al cilíndrico. Por todo ello, se han realizado simulaciones con ambos tipos de lastres con el objetivo de observar la importancia que pueden tener el peso y la geometría del contacto en las variables estudiadas.

Por otro lado, se modelaron inicialmente barandillas constituidas por redes de seguridad tipo $U$ sobre bastidores de tubo metálico con soportes rectos, de diversas escuadrías. Se han utilizado redes de $2 \mathrm{~m}$ de alto, por encima del mínimo normativo (1 $\mathrm{m})$, por considerar el riesgo de impactos directos contra la parte superior del bastidor, ya que con menor dimensión es posible que partes vitales del cuerpo lleguen a su altura en un evento de caída con rodadura por la superficie de trabajo. A partir de los resultados obtenidos para ellos, y estimadas las flechas generadas, se diseñaron otros soportes "ergonómicos" que permitieran el desarrollo de aquéllas, aún en el caso de caídas frente a soporte, sin impacto sobre éstos, con el fin de evitar la aparición de fuerzas mucho mayores contra estos elementos mucho más rígidos.

\subsection{Modelo numérico}

Para el desarrollo del modelo se han establecido los requisitos geométricos que limitan superiormente la clase $\mathrm{C}$ en UNE-EN 13374: ángulo de la superficie de trabajo inclinado $60^{\circ}$ con la horizontal, y $5 \mathrm{~m}$ de distancia recorrida por el lastre antes del impacto sobre la red.

Para diversas configuraciones, que se pormenorizan más adelante, se realizan las modelizaciones del sistema de protección con el programa de elementos finitos Ansys, utilizando perfiles metálicos tubulares de distintas secciones transversales como soporte de la red, con las siguientes características:

Red de cuadrícula $10 \times 10 \mathrm{~cm}^{2}$, peso de trencilla $0,008 \mathrm{Kg} / \mathrm{m}$.
Acero en soportes de un límite elástico de $240 \mathrm{~N} / \mathrm{mm}^{2}$.

En el fenómeno del impacto se generan grandes movimientos y giros en la estructura, tanto en los soportes como en la red; además en la red se Ilega a grandes deformaciones. Ésta tiene rigidez inicial transversal prácticamente nula, que va aumentando conforme se tensa, al menos en parte de ella. Se desconocen los puntos de contacto entre el cuerpo que cae y la red, puntos que van cambiando a lo largo del fenómeno y cuya identificación real afecta decisivamente a los esfuerzos generados. Los soportes metálicos presentan comportamiento elastoplástico, fácil de modelar en el acero como bilineal. La red presenta también un comportamiento elastoplástico mucho más complejo, que también tiene una importancia decisiva en relación con su capacidad de absorción de energía, una variable clave de este problema, y que repercute directamente en los soportes y en los esfuerzos sobre el accidentado (9).

Para tener en cuenta todas estas premisas, los elementos de Ansys utilizados para cada material son:

- Red (LINK10). El modelo propuesto para las trencillas de la red es una ley de comportamiento elástico bilineal sin resistencia a la compresión y con amortiguamiento estructural (Figura 2).

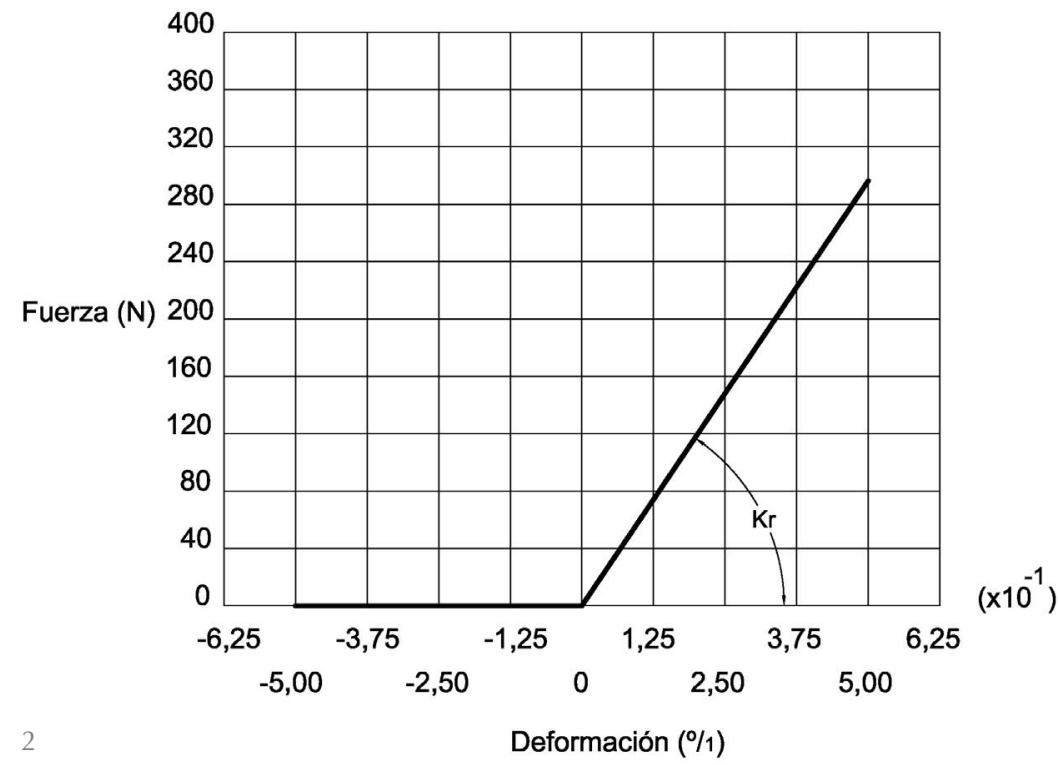

Los parámetros del comportamiento de la red para que ésta se comporte de forma equivalente a la observada realmente se toman de (9), los cuales se obtuvieron a partir de los trabajos teóricos y experimentales realizados en los años 90 para redes tipo $\mathrm{V}(8)$ y (9), y son: 
3. Posición de red vertical y perpendicular a la superficie de trabajo.

4. Soportes de barandillas sometidos a ensayos dinámicos.

$K r$ : Pendiente de la recta que representa un comportamiento proporcional de la red a tracción $(610 \mathrm{~N})$.

$\beta$ : Multiplicador de la matriz de rigidez con el que se obtiene la matriz de amortiguamiento estructural, cuyo valor es 0,34 .

La ley de comportamiento bilineal equivalente para la red se aleja de la real (5) principalmente por dos motivos:

- El apriete de los nudos.

- La relación entre tensión y deformación cuando la red trabaja a tracción no es una línea recta.

Conviene apuntar la prudencia en la credibilidad de algunos de los resultados obtenidos en esta modelización, que debería ser contrastada con ensayos experimentales para este tipo de red, pues se parte de los datos de rigidez y amortiguamiento contrastados para redes tipo $\mathrm{V}$.

- Lastre. Con él se define el elemento que lanzaremos sobre la red. Para el caso de la esfera se han utilizado elementos rígidos (MASS21). Para el caso del cilindro se ha modelado éste con dos tipos de elementos, interiormente con SHELL181 de material acero y un revestimiento exterior de caucho muy deformable, siempre conforme a (16) y (18), con SOLID45.

- Soporte o bastidor metálico (BEAM188). De esta forma podemos definir la geometría del mismo, en este caso la elegida es un perfil tubular hueco de diferentes diámetros y espesores. Con este modelo podemos obtener movimientos y tensiones en el bastidor.
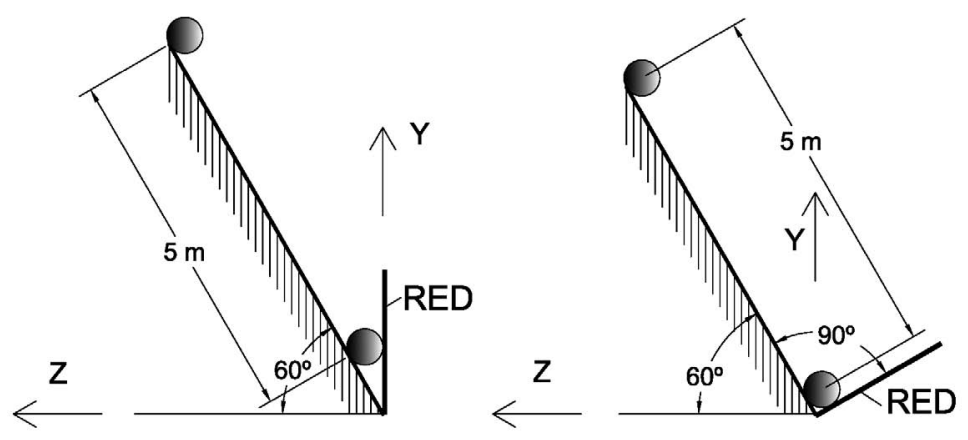

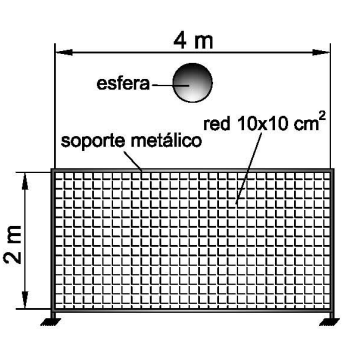

Alzado soporte recto

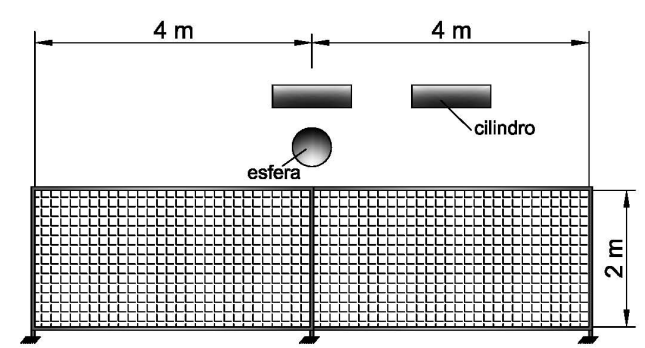

Alzado soporte ergonómico
- Elementos de contacto (CONTA), (TARGET) (9): Se ha definido el contacto entre el lastre y la red como un contacto variable entre dos superficies, una rígida (lastre) y la otra deformable (red).

Como la herramienta informática utilizada no permite definir los elementos de contacto directamente sobre los elementos lineales de la red, sino que deben definirse sobre elementos superficiales, se han utilizado unos elementos superficiales auxiliares de masa y rigidez despreciables que sólo transmiten esfuerzos de membrana de tracción y que tienen por nudos los de la red.

La rigidez del contacto se actualiza en cada instante analizado y cada punto de contacto a partir de la tensión media del elemento involucrado en ese punto, de manera que la rigidez no es tan pequeña que provoque sobre-penetración ni tan grande que cause divergencias en el análisis.

Para cada caso se obtienen:

- Los desplazamientos del lastre, red y soporte.

- La aceleración que sufre el lastre en su desplazamiento, variable relacionada con los máximos esfuerzos que sufre el accidentado.

- Las tensiones en los soportes y los axiles en las trencillas de la red, así como su deformación unitaria.

\subsection{Planteamiento con soporte recto y lastre esférico}

Corresponde a los primeros modelos analizados, para la simulación de un accidentado golpeando con la cabeza o los pies contra la protección.

Se analizan dos posibles posiciones de la red de protección, siguiendo las especificaciones establecidas en UNE- EN 13.374 (Figura 3), pero con el lastre esférico anteriormente descrito.

En ambas posiciones, suponemos un sistema de protección de borde tipo $\mathrm{C}$, formado por un bastidor metálico tubular de sección constante para montantes y largueros, en cuyo interior se sujeta, solamente a éstos últimos, una red de $4 \times 2 \mathrm{~m}^{2}$; el bastidor se sujeta únicamente en los extremos inferiores, tal y como se observa en la Figura 4, donde también se esquematizan las posiciones de lanzamiento de lastres, a centro de paño entre soportes y frente al soporte central. 


\subsection{Planteamiento con soporte ergonómico y lastre esférico}

A continuación se procedió a la sustitución del soporte recto por uno ergonómico (ahora con límite elástico de 275 N/ $\mathrm{mm}^{2}$ ), solucionando el problema de los puntos duros del sistema de protección en los soportes rectos del planteamiento en el apartado 2.2, aportando así, una solución al citado problema que dejaba planteado la vigente versión de UNE-EN 13374, en su apartado 6.4.3. Su diseño está basado en la deformación previa de la red en el espacio necesario para que se detenga el lastre sin alcanzar el perfil del soporte, evaluado en la primera serie de simulaciones.

\subsection{Planteamiento con soporte ergonómico y lastre cilíndrico}

Se usa para simulación de un impacto lateral del accidentado sobre la red de la protección y es el ensayo establecido por UNE-EN 13374.

Se lleva a cabo con el lastre cilíndrico de $30 \mathrm{~cm}$ de diámetro, $100 \mathrm{~cm}$ de largo y $75 \mathrm{Kg}$ de peso, según (16) y (18), que caerá sobre la red.

Los elementos de Ansys utilizados son los indicados en el punto 2.1.

\subsubsection{Lanzamiento frente a soporte central}

Mediante el modelo de elementos finitos Ansys se realizan lanzamientos del cilindro frente al soporte central, sobre una red de $8 \mathrm{~m}$ de largo y $2 \mathrm{~m}$ de alto, sujeta por tres soportes separados $4 \mathrm{~m}$ con pasamanos superior y rodapié inferior.

Se analizan distintas posiciones de la red, desde su posición vertical hasta su posición horizontal, analizando también dos posiciones intermedias (Figura 5):

- RED $0^{\circ}$ (vertical).

- RED $30^{\circ}$.

- RED $60^{\circ}$ (perpendicular a la superficie de trabajo).

- RED $90^{\circ}$ (horizontal).
Para todos estos casos, se estudia el comportamiento del sistema en cuanto a la aceleración máxima sufrida por el lastre, flecha, axil y deformación máxima de la red y tensiones del soporte.

Con los lanzamientos frente a soporte, comprobamos si éste es alcanzado por el lastre, con la finalidad de resolver los puntos duros que ellos suponen.

\subsubsection{Lanzamiento entre soportes ergonómicos en posición red $60^{\circ}$ (perpendicular)}

En este supuesto, se comprueba el sistema para la recepción del lastre a la mitad de distancia entre dos soportes. Esta disposición resulta previsiblemente más desfavorable para la capacidad resistente del sistema, al sufrir los soportes cercanos al lastre y los largueros una flexión lateral, lo que influirá notablemente en las tensiones sufridas por éstos.

Sólo se analiza la posición perpendicular para comparar los resultados obtenidos con esa misma posición, pero con lanzamiento frente a soportes, del apartado anterior.

Esta comparativa será extrapolable para el resto de las posiciones analizadas de forma directa.

\subsection{Discusión de la flecha mínima requerida}

Finalmente, para valorar el requisito de flecha mínima establecido por UNE-EN 13374, se ha analizado un conjunto de soportes ergonómicos, con bastidor de perfil tubular $80 \times 3$ con redes de rigidez creciente hasta disminuir la flecha máxima a los 200 $\mathrm{mm}$ establecidos, midiendo las aceleraciones impuestas al lastre.

Paralelamente se realiza un estudio analítico puramente cinemático, inspirado cualitativamente en las deceleraciones de los resultados numéricos, que permitirá corroborar los relativos a flecha mínima y factor de impacto y, a través de una fórmula simple, discutir el valor actual para dicha flecha y proponer valores más razonables.
5. Alzado lateral para distintas posiciones de la red.

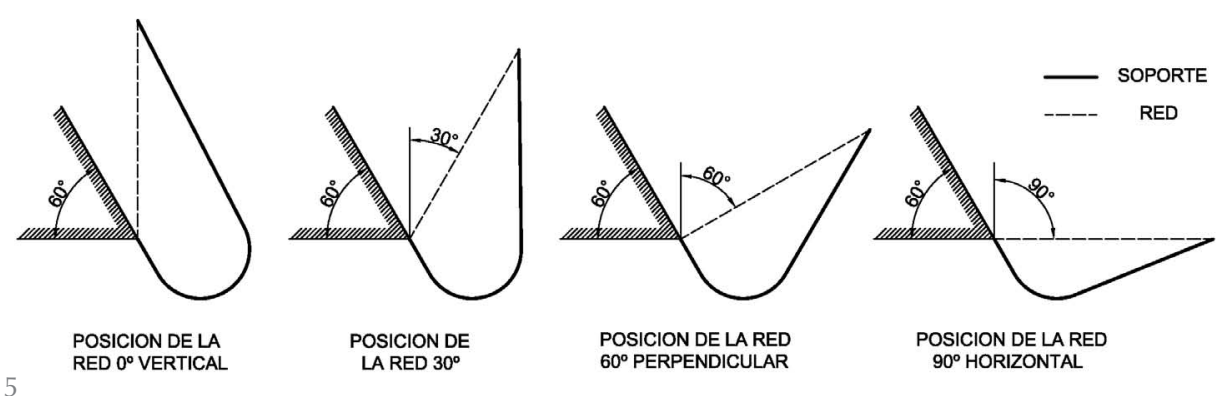


Tabla 1. Resultados para distintas disposiciones de soportes y redes, con lastre esférico

\begin{tabular}{|c|c|c|c|c|c|c|c|c|c|c|c|c|c|c|c|}
\hline \multirow{3}{*}{$\begin{array}{c}\begin{array}{c}\text { Seccion } \\
\text { bastidor }\end{array} \\
\\
\varnothing \times t \\
(\mathrm{~mm})\end{array}$} & \multicolumn{5}{|c|}{$\begin{array}{c}\text { Soporte recto, } \\
\text { lastre esferico entre soportes }\end{array}$} & \multicolumn{5}{|c|}{$\begin{array}{l}\text { Soporte recto, } \\
\text { lastre esferico entre soportes }\end{array}$} & \multicolumn{5}{|c|}{$\begin{array}{l}\text { Soporte ergonómico, } \\
\text { lastre esférico frentea soporte }\end{array}$} \\
\hline & \multirow[b]{2}{*}{ 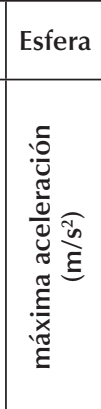 } & \multicolumn{3}{|c|}{$\begin{array}{l}\text { Red vertical } \\
4 \times 2 \mathrm{~m}^{2}\end{array}$} & \multirow[b]{2}{*}{ 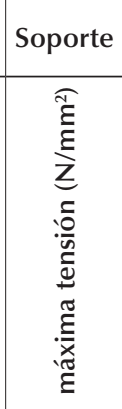 } & \multirow[b]{2}{*}{ 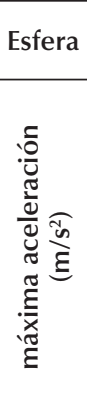 } & \multicolumn{3}{|c|}{$\begin{array}{c}\text { Red Perpendicular } \\
4 \mathrm{X} 2 \mathrm{~m}^{2}\end{array}$} & \multirow[b]{2}{*}{ 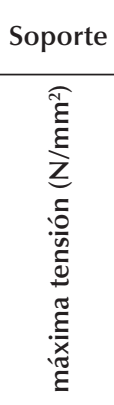 } & \multirow[b]{2}{*}{ 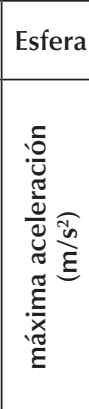 } & \multicolumn{3}{|c|}{$\begin{array}{l}\text { Red Perpendicular } \\
8 \times 2 \mathrm{~m}^{2}\end{array}$} & \multirow[b]{2}{*}{ 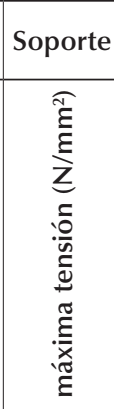 } \\
\hline & & 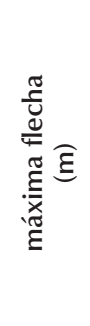 & 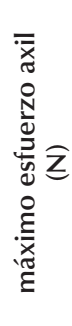 & 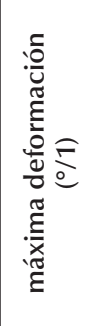 & & & 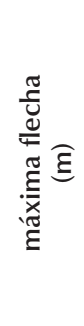 & 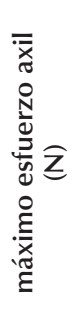 & 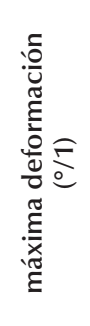 & & & 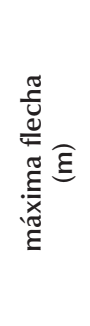 & 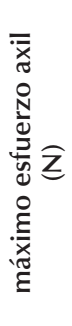 & 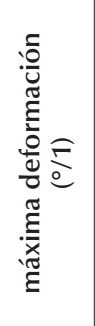 & \\
\hline $40 \times 2$ & $-90,2$ & 1,068 & 895 & 1,468 & 238 & $-56,0$ & 1,315 & 500 & 0,820 & 238 & $-52,2$ & 1,320 & 475 & 0,778 & 273 \\
\hline $40 \times 3$ & $-110,0$ & 0,955 & 836 & 1,37 & 2 & $-62,1$ & 1,181 & 569 & 0,933 & 237 & $-57,7$ & 180 & 553 & 907 & 272 \\
\hline $50 \times 2$ & $-110,6$ & 0,933 & 820 & 1,345 & 236 & $-64,5$ & 1,142 & 589 & 0,966 & 237 & $-59,6$ & 1,130 & 580 & 0,952 & 272 \\
\hline $50 \times 3$ & $-126,7$ & 0,862 & 809 & 1.326 & 235 & $-67,0$ & 1,084 & 612 & 1,003 & 236 & $-64,4$ & 1,063 & 602 & 0,986 & 271 \\
\hline $60 \times 2$ & $-125,0$ & 0,887 & 804 & 1,319 & 240 & $-67,1$ & 1,080 & 615 & 1,009 & 240 & $-65,5$ & 1,050 & 596 & 0,976 & 271 \\
\hline $60 \times 3$ & 32,0 & 0,854 & 791 & 1,297 & 240 & $-68,0$ & 1,032 & 627 & 1,028 & 240 & & & & & \\
\hline $60 \times 4$ & & & & & & $-68,5$ & 1,008 & 634 & 1,040 & 203 & & & & & \\
\hline $80 \times 2$ & & & & & & $-68,7$ & 0,996 & 634 & 1,039 & 215 & & & & & \\
\hline $80 \times 3$ & & & & & & $-69,5$ & 0,983 & 634 & 1,039 & 146 & & & & & \\
\hline $80 \times 4$ & & & & & & $-69,6$ & 0,978 & 633 & 1,038 & 120 & & & & & \\
\hline
\end{tabular}

Tabla 2. Resultados para distintos ángulos de posición de la red, con soporte ergonómico y lastre cilíndrico cayendo frente al soporte central

\begin{tabular}{|c|c|c|c|c|c|c|c|c|c|c|c|c|c|c|c|c|c|c|c|c|}
\hline \multirow{3}{*}{\begin{tabular}{|c|}
$\begin{array}{l}\text { Sección } \\
\text { bastidor }\end{array}$ \\
\\
$\begin{array}{l}\varnothing \times \mathrm{t} \\
(\mathrm{mm})\end{array}$ \\
\end{tabular}} & \multicolumn{5}{|c|}{$\begin{array}{l}\text { Lastre cilindro, frente soporte } \\
\text { ergonómico central }\end{array}$} & \multicolumn{5}{|c|}{$\begin{array}{l}\text { Lastre cilindro, frente soporte } \\
\text { ergonómico central }\end{array}$} & \multicolumn{5}{|c|}{$\begin{array}{l}\text { Lastre cilindro, frente soporte } \\
\text { ergonómico central }\end{array}$} & \multicolumn{5}{|c|}{$\begin{array}{l}\text { Lastre cilindro, frente soporte } \\
\text { ergonómico central }\end{array}$} \\
\hline & \multirow{2}{*}{ 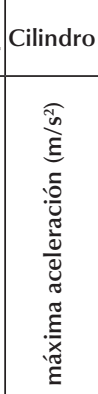 } & \multicolumn{3}{|c|}{$\begin{array}{l}\text { Red } 8 \times 2 \mathrm{~m}^{2} \\
0^{\circ}(\text { vertical })\end{array}$} & \multirow[b]{2}{*}{ 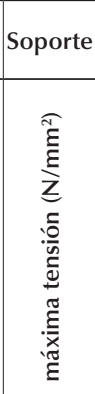 } & \multirow[b]{2}{*}{ 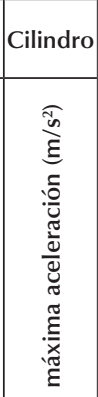 } & \multicolumn{3}{|c|}{$\begin{array}{c}\operatorname{Red} 8 \times 2 \mathrm{~m}^{2} \\
30^{\circ}\end{array}$} & \multirow[b]{2}{*}{ 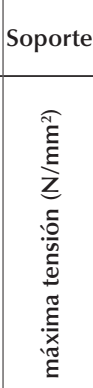 } & \multirow[b]{2}{*}{ 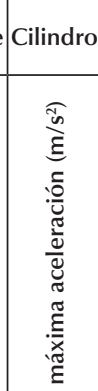 } & \multicolumn{3}{|c|}{$\begin{array}{c}\text { Red } 8 \times 2 \mathrm{~m}^{2} \\
60^{\circ} \text { (perpendicular) }\end{array}$} & \multirow[b]{2}{*}{ 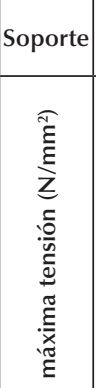 } & \multirow[b]{2}{*}{ 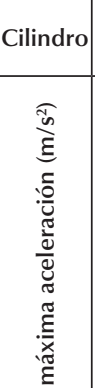 } & \multicolumn{3}{|c|}{$\begin{array}{c}\text { Red } 8 \times 2 \mathrm{~m}^{2} \\
90^{\circ} \text { (horizontal) }\end{array}$} & \multirow[b]{2}{*}{ 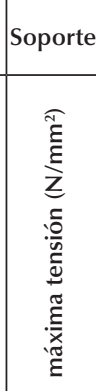 } \\
\hline & & 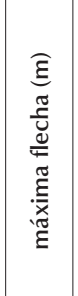 & 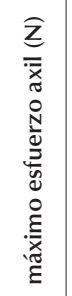 & 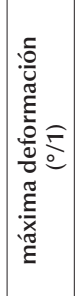 & & & 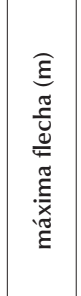 & 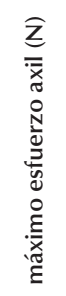 & 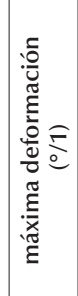 & & & 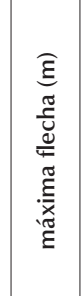 & 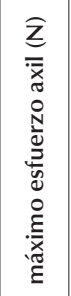 & 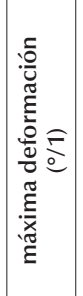 & & & 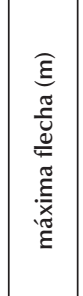 & 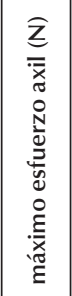 & 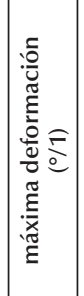 & \\
\hline $25 \times 2$ & 30,6 & & & & & & & & $0, \angle 00$ & & & & & & & & & & & \\
\hline $25 x$ & 3 & 3 & 208 & 0,341 & 27 & 9 & 0,920 & 199 & 0,32 & & & {$[1,035$} & & & & & & & & \\
\hline $30 \times 2$ & $-142,8$ & 0,777 & 230 & 0,378 & 272 & $-112,9$ & 0,885 & 217 & 0,356 & 269 & $-7 x$ & 0,997 & 1 & 0,3 & 27 & & & & & \\
\hline $30 \times 3$ & $-158,3$ & 0,673 & 250 & 0,410 & 271 & $-126,4$ & 0,754 & 243 & 0,398 & 27 & 3 & 0,841 & 224,6 & 0,3 & 27 & & & & & \\
\hline $35 \times 2$ & 5,6 & 0 & 252 & 0,414 & 272 & 9 & 0 & 2 & 0 & 2 & 2 & 39 & 227 & 0 & 273 & 0 & 0,913 & 367 & c & 277 \\
\hline $35 \times 3$ & 1,1 & 8 & 268 & 0,439 & 271 & 4 & 4 & 264 & 0 & & J & 1 & 1 & & & O & $0,104]$ & 372 & c & \\
\hline $40 \times 2$ & 72,9 & 618 & 267 & 0,438 & 271 & $-133,7$ & 0,679 & 265 & 0,434 & 26 & $-85,7$ & 0,731 & 5 & 0, & & & 0,785 & 66 & 0,2 & 27 \\
\hline $40 \times 3$ & $-189,7$ & 0,554 & 279 & 0,458 & 271 & $-145,1$ & 0,600 & 285 & 0,467 & 27 & $-95,8$ & 0,641 & 274,3 & 0,4 & 243 & y & 0,720 & 314 & 0,3 & 270 \\
\hline $45 \times 2$ & 0,9 & 4 & 277 & 0,4 & 271 & 7 & 3 & 283 & 0 & 268 & $-c$ & 4 & 26 & 0 & 218 & 8 & 0,7 & 6 & 1 & 7 \\
\hline $45 \times 3$ & $-196,6$ & 0,530 & 282 & 0,463 & 271 & & 0,565 & 295 & 0,484 & & $-99,9$ & 0,617 & 278,5 & & & & 0,686 & 273 & 0,355 & 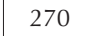 \\
\hline $50 \times 2$ & $-189,4$ & 0,542 & 281 & 0,461 & 271 & $-149,8$ & 0,577 & 292 & 0,478 & 263 & $-99,5$ & 0,623 & 279,5 & 0,458 & 199 & $-57,9$ & 0,685 & 265 & 0,366 & 270 \\
\hline $50 \times 3$ & $-209,8$ & 0,502 & 282 & 0,462 & 270 & $-161,1$ & 0,534 & 308 & 0,505 & 259 & $-104,7$ & 0,589 & 288,9 & 0,47 & 18 & $-58,8$ & 0,669 & 245 & 0,373 & 270 \\
\hline $55 \times 2$ & $-201,8$ & 507 & 280 & 0,459 & 271 & $-156,0$ & 0,503 & 299 & 0,490 & 25 & -10 & 0,596 & 2 & 0,4 & 10 & 9 & 0,672 & 51 & 0,3 & 270 \\
\hline $55 \times 3$ & $-212,7$ & 22 & 274,9 & 0,450 & 27 & 9 & 18 & 310 & 0 & 212 & 0 & ] & 2 & of & 185 & & 0,000 & 238 & 71 & \\
\hline $60 \times 2$ & $-212,3$ & 0,494 & 283 & 0,463 & 269 & $-162,7$ & 0,530 & 307 & 0,504 & & 3,8 & 583 & , & 10 & & 9,2 & 0,665 & 35 & 07 & 7 \\
\hline $60 \times 3$ & $-215,2$ & 0,465 & 278 & 0,456 & 262 & \begin{tabular}{|l|}
$-166,2$ \\
\end{tabular} & 0,495 & 313 & 0,513 & 213 & $-111,1$ & 0,559 & 295,3 & 0,484 & 178 & $-59,2$ & 0,658 & 226 & 0,370 & 257 \\
\hline
\end{tabular}




\section{RESULTADOS}

\subsection{Soporte recto y lastre esférico}

En los dos primeros cuadros de la Tabla 1 se recogen los resultados para posición de red vertical y perpendicular a la superficie de trabajo, con caída de la esfera al centro del paño entre soportes.

Las aceleraciones sufridas por el lastre en posición vertical de la red son mayores que para la posición perpendicular de la red. Este resultado es importante, dado que dicha aceleración facilita directamente la fuerza máxima sufrida por el accidentado. Como se observa, para una misma sección de soporte $(60 \times 2)$ obtenemos unas aceleraciones de $125 \mathrm{~m} / \mathrm{s}^{2}(12,5 \cdot \mathrm{g})$ y $67,1 \mathrm{~m} / \mathrm{s}^{2}$ $(6,7 \cdot g)$ en posición vertical y perpendicular de la red, respectivamente.

La flecha generada en la red es mayor en la posición perpendicular, alrededor de un $17 \%$ de media más que en la posición vertical. La posición perpendicular tendrá, por consiguiente, un comportamiento mucho más flexible para el accidentado.

Los máximos esfuerzos axiles para las trencillas de la red están en torno a los $800 \mathrm{~N}$ para la posición vertical y $600 \mathrm{~N}$ para la posición perpendicular.

Las deformaciones de la red son también mayores en la posición vertical que en la perpendicular. Los resultados obtenidos indican que para los soportes de menor escuadría se alcanzan las tensiones de plastificación. Estas tensiones disminuyen a medida que aumenta la sección del perfil, hasta mantenerse dentro del rango elástico para las mayores escuadrías.

\subsection{Soporte ergonómico y lastre esférico}

El tercer cuadro de la Tabla 1 recoge los resultados para el caso de esfera cayendo frente al soporte central.

Se obtiene una flecha de la red superior a $1 \mathrm{~m}$, en todas las secciones analizadas. Las tensiones máximas de los soportes superan los $270 \mathrm{~N} / \mathrm{mm}^{2}$, alcanzando la plastificación. En cuanto a las aceleraciones sufridas por el lastre, éstas Ilegan hasta los $65,5 \mathrm{~m} / \mathrm{s}^{2}$ para los soportes más rígidos analizados.

\subsection{Soporte ergonómico y lastre cilíndrico}

\subsubsection{Lanzamiento frente a soporte centra}

En la Tabla 2 se resumen los valores obtenidos en cuanto a aceleraciones sufridas por el lastre, flecha, axil y deformación de la red, tensiones de los soportes originales ergonómicos de distintas escuadrías, para la posición perpendicular de la red y lastre cilíndrico cayendo frente al soporte central.

\subsubsection{RED $0^{\circ}$ (vertical)}

Las aceleraciones sufridas por el lastre oscilan entre 130 y $215 \mathrm{~m} / \mathrm{s}^{2}$ (factor de impacto entre 13 y 21,5$)$.

La flecha máxima de la red es de 0,93 m para el soporte más delgado, siendo de 0,465 m para la sección mayor.

En cuanto a las tensiones, todos los soportes Ilegan hasta la plastificación excepto los dos más grandes $(60 \times 2$ y 60×3).

\subsubsection{RED $30^{\circ}$}

Las aceleraciones sufridas por el lastre oscilan entre 99 y $166 \mathrm{~m} / \mathrm{s}^{2}$. Es decir, factores de impacto entre 10 y 16,6 .

La flecha máxima de la red es de 1,090 m para el soporte más delgado, siendo de 0,495 m para la sección mayor.

En cuanto a las tensiones, sólo llegan a la plastificación los seis soportes de menor sección, no superando los $213 \mathrm{~N} / \mathrm{mm}^{2}$ para el de sección mayor (60×3).

\subsubsection{RED $60^{\circ}$ (perpendicular)}

Las aceleraciones sufridas por el lastre están entre 70 y $111 \mathrm{~m} / \mathrm{s}^{2}$, lo que supone un factor de impacto de 7 y 11 , respectivamente.

Los axiles de la red van en aumento a medida que aumenta la rigidez del soporte, de $173 \mathrm{~N}$ a $295 \mathrm{~N}$ para los soportes de mayor diámetro.

Las deformaciones de la red aumentan a medida que lo hace la rigidez del soporte. Estabilizándose en 0,484 para las secciones mayores. Las flechas máximas oscilan entre 1,05 y 0,56 m (Figuras 6 y 7 ).

Las tensiones de los soportes delgados, son mayores, llegando a la plastificación, y disminuyen a medida que aumentamos la sección de los soportes hasta quedar dentro del rango elástico.

\subsubsection{RED $90^{\circ}$ (horizontal)}

Las aceleraciones sufridas por el lastre están entre 48 y $59 \mathrm{~m} / \mathrm{s}^{2}$, lo que supone un factor de impacto de 4,8 y 5,9 , respectivamente.

Las tensiones de los soportes delgados, son mayores, Ilegando a la plastificación, y dis- 

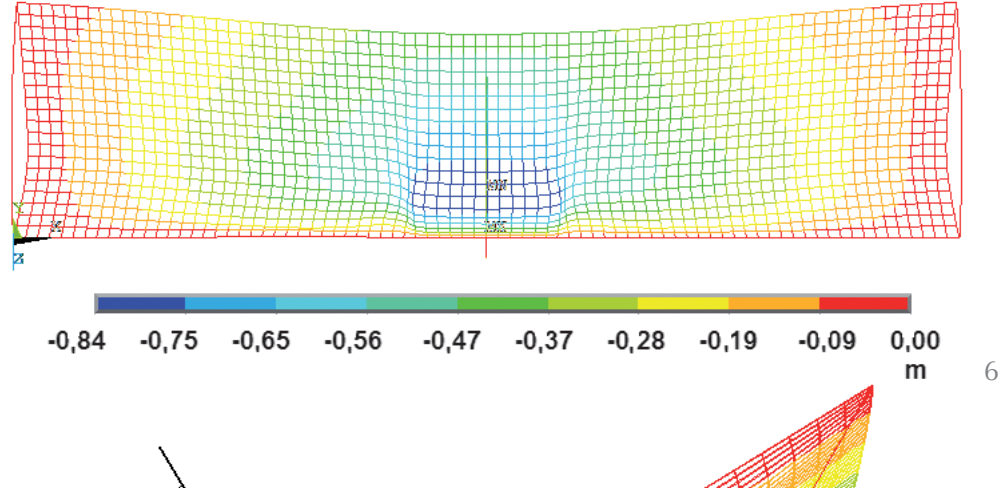

0,84

$-0,75 \quad-0,65 \quad-0,56$

$-0,4$

$-0,37$

6. Vista frontal de la red perpendicular con máxima flecha.

7. Vista lateral de la red perpendicular con máxima flecha.

8. Vista de la red en posición horizontal en distintos instantes de tiempo. minuyen a medida que aumentamos la sección de los soportes hasta quedar dentro del rango elástico con las secciones más grandes.

La flecha de la red oscila entre 0,913 m para el soporte de menor diámetro y 0,658 m para el soporte más rígido de los estudiados (60×3).

Para esta disposición se observa (Figura 8) un tiempo de retención más dilatado, al tener el lastre un recorrido más largo sobre la red, lo cual justifica las menores deceleraciones registradas. Sin embargo, el lastre se acerca peligrosamente al pasamanos, con riesgo de impacto sobre el mismo, e incluso de caída al vacío.

\subsubsection{Lanzamiento entre soportes en posición red $60^{\circ}$ (perpendicular)}

En la Tabla 3 se resumen los valores obtenidos en cuanto a aceleraciones sufridas por el lastre, flecha, axil y deformación de la red, tensiones de los soportes originales ergonómicos de distintas escuadrías, para la posición perpendicular de la red y lastre cilíndrico lanzado al centro del paño entre dos soportes.
Tabla 3.

Resultados para lanzamiento entre soportes

\begin{tabular}{|c|c|c|c|c|c|}
\hline \multicolumn{6}{|c|}{ Lastre cilindro entre soportes ergonómicos } \\
\hline $\begin{array}{l}\text { Sección } \\
\text { bastidor }\end{array}$ & Cilindro & $\begin{array}{r}\mathrm{Re} \\
60^{\circ}(\mathrm{pe}\end{array}$ & beno & $\begin{array}{l}a^{2} \\
\text { cular) }\end{array}$ & Soporte \\
\hline$\varnothing x t(m m)$ & 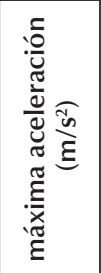 & 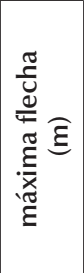 & 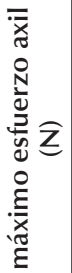 & 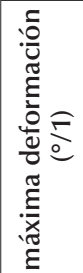 & 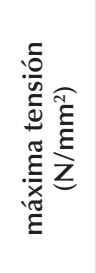 \\
\hline $25 \times 3$ & $-53,9$ & 1,267 & 215 & 0,352 & 271 \\
\hline $30 \times 2$ & $-56,5$ & 1,203 & 231 & 0,379 & 270 \\
\hline $30 \times 3$ & $-65,8$ & 1,000 & 256 & 0,420 & 270 \\
\hline $35 \times 2$ & $-66,6$ & 1,000 & 256 & 0,420 & 270 \\
\hline $35 \times 3$ & $-77,5$ & 0,850 & 279 & 0,457 & 270 \\
\hline $40 \times 2$ & $-77,1$ & 0,865 & 279 & 0,458 & 270 \\
\hline $40 \times 3$ & $-87,2$ & 0,749 & 292 & 0,479 & 270 \\
\hline $45 \times 2$ & $-85,7$ & 0,774 & 291 & 0,477 & 270 \\
\hline $45 \times 3$ & $-96,1$ & 0,695 & 300 & 0,492 & 270 \\
\hline $50 \times 2$ & $-95,0$ & 0,714 & 298 & 0,488 & 270 \\
\hline $50 \times 3$ & $-101,6$ & 0,654 & 304 & 0,498 & 265 \\
\hline $55 \times 2$ & $-86,9$ & 0,680 & 301 & 0,494 & 270 \\
\hline $55 \times 3$ & $-104,8$ & 0,631 & 307 & 0,503 & 250 \\
\hline $60 \times 2$ & $-104,3$ & 0,645 & 307 & 0,504 & 258 \\
\hline $60 \times 3$ & $-106,4$ & 0,620 & 308 & 0,505 & 238 \\
\hline
\end{tabular}

Las aceleraciones sufridas por el lastre están entre 53,9 y $106,4 \mathrm{~m} / \mathrm{s}^{2}$, lo que supone un factor de impacto de 5,39 y 10,64 , respectivamente.

Los axiles de la red van en aumento a medida que aumenta la rigidez del soporte, de $215 \mathrm{~N}$ a $308 \mathrm{~N}$ para los soportes de mayor diámetro.

Las deformaciones de la red aumentan a medida que lo hace la rigidez del soporte. Estabilizándose en 0,505 para las secciones mayores.

Las tensiones de los soportes delgados, son mayores, llegando a la plastificación, y disminuyen a medida que aumentamos la sección de los soportes hasta quedar dentro del rango elástico, con $238 \mathrm{~N} / \mathrm{mm}^{2}$ para el de mayor diámetro $(60 \times 3)$.

\subsection{Flecha mínima y factor de impacto}

Con la modelización de Ansys del sistema provisional de protección de borde clase C, se ha comparado el mismo soporte ergo-

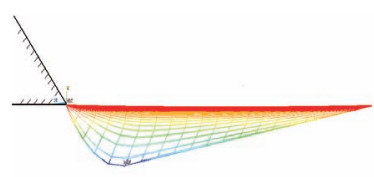

$t=6,15 s$
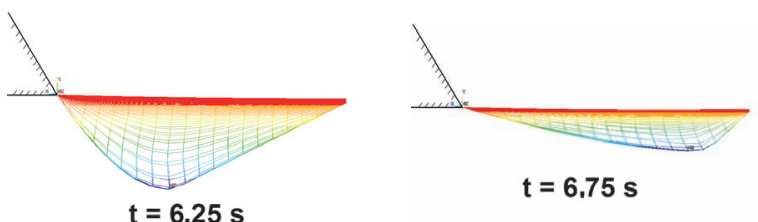
nómico de sección 80x3mm en posición perpendicular, para distintas rigideces de la red, obteniendo resultados de relevancia en cuanto al factor de impacto sufrido por el accidentado.

Para rigideces 10 veces superiores a la calibrada para el comportamiento real en redes tipo $\mathrm{V}$ (9), la flecha máxima se reduce prácticamente a los $200 \mathrm{~mm}$ establecidos como mínimo por UNE-EN 13374. Pero las aceleraciones correspondientes aumentan hasta valores superiores a $29 \mathrm{~g}$, los cuales son absolutamente inadmisibles para un accidentado que cayera sobre un sistema de protección de tal rigidez.

Independientemente de la precisión en el modelado de la rigidez de la red, la deceleración máxima a está directamente ligada a la velocidad inicial en el contacto $v_{0}$ y a la flecha máxima $f$. Considerando el movimiento desde el contacto con una aceleración bilineal (Figura 9) con la tipología observada en las simulaciones (Figura 10) puede obtenerse una fórmula simple [1] que relaciona con precisión aceptable el factor de impacto $\gamma$, la flecha máxima $f$ y la energía cinética a absorber ( $v_{0}$ por pérdida de cota $h$ durante la caída).

En efecto:

$$
\begin{aligned}
& a_{1}(t)=-\frac{a}{\Delta} t \Rightarrow v_{1}(t)=-\frac{a}{\Delta} \frac{t^{2}}{2}+v_{0} \Rightarrow s_{1}(t)=v_{0} t-\frac{a}{6 \Delta} t^{3}+0 \\
& a_{2}(t)=-a+\frac{t-\Delta}{2 \Delta} a \Rightarrow v_{2}(t)=\frac{a}{2 \Delta} \frac{t^{2}}{2}-\frac{3 a}{2} t+A \Rightarrow s_{2}(t)=A t-\frac{3 a}{4} t^{2}+\frac{a}{12 \Delta} t^{3}+B \\
& v_{2}(\Delta)=v_{1}(\Delta) \Rightarrow A=v_{0}+\frac{3}{4} a \Delta \\
& s_{2}(\Delta)=s_{1}(\Delta) \Rightarrow B=-\frac{a \Delta^{2}}{4} \\
& v_{2}(3 \Delta)=0 \Rightarrow \Delta=\frac{2 v_{0}}{3 a} \\
& f=s_{2}(3 \Delta)=\frac{8}{9} \frac{v_{0}^{2}}{a}=\frac{8}{9} \frac{2 g h}{a}=\frac{16}{9} \frac{h g}{a} \Rightarrow a=\frac{16}{9} \frac{h}{f} g=\gamma g \Rightarrow \\
& \gamma=1,77 \frac{h}{f}
\end{aligned}
$$

Con esta estimación, para la altura de caída del ensayo $h=5 \operatorname{sen} 60=4,33 \mathrm{~m}$, y la flecha mínima establecida por (18), $f=0,2 \mathrm{~m}$, se tendría $\gamma=38$. Estos valores corresponden, como se ha indicado, a los parámetros de masa y altura de caída del ensayo, que corresponderían, despreciando las pérdidas durante el deslizamiento o rodadura, a una energía de $\left(\mathrm{g} \approx 10 \mathrm{~m} / \mathrm{s}^{2}\right)$ :

$$
750 \mathrm{~N} \times 5 \operatorname{sen} 60 \mathrm{~m}=3248 \mathrm{~J}
$$

mayor que los $2200 \mathrm{~J}$ establecidos por la propia norma (apartado 6.4.3) para baran-
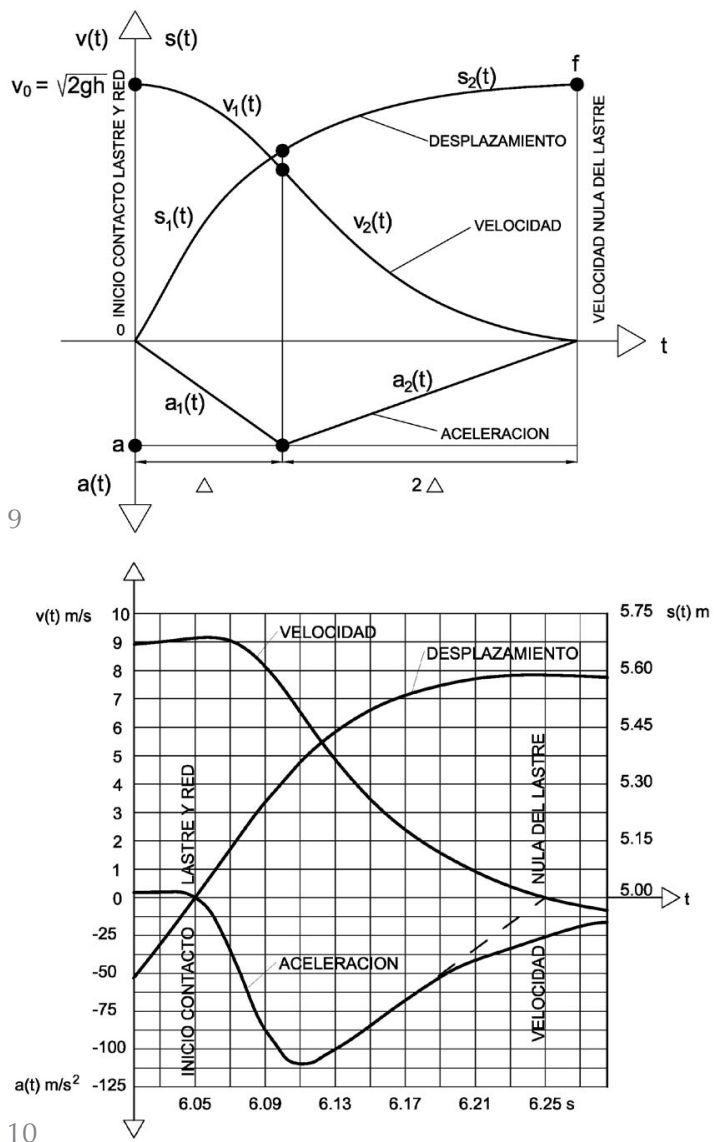

9. Aceleración bilineal simplificada, Velocidad y Desplazamiento del lastre tras impactar en la red.

10. Aceleración, Velocidad y Desplazamiento del lastre tras impactar en la red, según el modelo numérico con elementos finitos. 
para 3248 J y $75 \mathrm{Kg}$

$$
\gamma=1,77 \frac{433}{f} \approx 6 \Rightarrow f \approx 128 \mathrm{~cm}
$$

y para 2200 J y $75 \mathrm{Kg}$

$$
\gamma=1,77 \frac{290}{f} \approx 6 \Rightarrow f \approx 86 \mathrm{~cm}
$$

Los valores así obtenidos concuerdan sensiblemente con las aceleraciones y flechas máximas que los modelos numéricos suministran para red perpendicular a la superficie de trabajo (Tablas 2 y 3 con red $60^{\circ}$ perpendicular).

Estas consideraciones ponen de manifiesto la conveniencia de aumentar considerablemente los valores de flecha mínima requeridos para barandillas tipo $\mathrm{C}$ y tipo $\mathrm{B}$ por UNE-EN 13374, en su próxima revisión.

\section{CONCLUSIONES}

1. El ángulo entre la red y la superficie de trabajo influye de forma importante en los esfuerzos sobre el sistema y el factor de impacto que sufre el accidentado. Ángulos menores generan mayores esfuerzos y coeficientes de impacto, y viceversa; si bien con ángulos muy grandes el recorrido del accidentado hasta su detención llega hasta el borde superior del bastidor generando riesgo de impacto directo o incluso de sobrepasar el sistema y caer al vacío. De acuerdo con ello, la posición vertical del plano del sistema, admitida en UNE-EN13374 debería ser revisada, al menos para las barandillas clase $\mathrm{C}$, a favor de una inclinación más próxima a la normal a la superficie de trabajo.

2. El uso de soportes cuya directriz permita el desarrollo de las deformaciones de la red sin llegar a impactar directamente con el accidentado, aquí denominados ergonómicos, resuelve satisfactoriamente el problema de los impactos directos sobre partes demasiado rígidas, capaces de generar daños inadmisibles al accidentado, y asunto pendiente en el actual texto normativo.

3. Tanto para impacto a centro de paño como frente a soporte, los contactos de cabeza o de pies (lastre esférico) generan mayores flechas y menores factores de impacto que los contactos laterales (lastre cilíndrico), en ambos casos con una proporción aproximada de 3 a 2 . Sin embargo, las máximas solicitaciones en las trencillas de la red son casi el doble para el lastre esférico, como consecuencia de su efecto más localizado.
4. En cualquier caso, la caída lejos de la zona de soportes resulta menos dura para el accidentado y más para el sistema, aumentando la solicitación del bastidor. Salvo para las escuadrías más grandes, en éste último se alcanza la plastificación, siendo su extensión considerable para las escuadrías menores analizadas, si bien ello redunda en una disminución notable del factor de impacto al aumentar las flechas máximas.

5. El factor de impacto que sufre el accidentado tiene relación directa con la velocidad alcanzada (energía potencial perdida) durante la caída y con la flecha máxima del sistema durante la retención. La expresión simple [1] obtenida en el artículo, que relaciona estas variables, permite tomar decisiones de diseño con precisión aceptable y sus predicciones concuerdan bien con los resultados del modelo numérico.

6. Una conclusión importante derivada de la anterior es que para tener factores de impacto razonablemente bajos, se requiere el desarrollo de flechas considerables durante la retención. Los valores mínimos establecidos por la actual UNE-EN 13374, $200 \mathrm{~mm}$ para clase C y $100 \mathrm{~mm}$ para clase $\mathrm{B}$, deberían revisarse al alza hasta valores del orden de $1 \mathrm{~m}$ para la primera y $0,5 \mathrm{~m}$ para la segunda.

7. A pesar de que la validez de algunas de las conclusiones anteriores es independiente de los parámetros utilizados para describir el comportamiento de la red, sería conveniente realizar ensayos reales instrumentados para verificar la validez de aquéllos, sólo contrastados para redes tipo $\mathrm{V}$.

8. Una cuestión importante que también podría dar lugar a mejoras en el actual texto normativo es el análisis de la retención con barandillas de $1 \mathrm{~m}$ de alto (el mínimo actual) ya que éstas, además del riesgo citado de impacto contra su borde superior más duro, y debido a la menor longitud de las trencillas verticales de la red, pueden resultar considerablemente más rígidas que las analizadas en el artículo (alto de $2 \mathrm{~m}$ ), y por tanto, conducir a factores de impacto inadmisibles con la misma escuadría en el bastidor.

9. En relación con la anterior, también sería conveniente analizar el comportamiento de las redes dispuestas al rombo (en lugar de al cuadro, analizadas en este artículo) ya que la mayor longitud de las alineaciones de trencillas puede 
paliar la mayor rigidez de las redes menos anchas.

10. Finalmente, queda también pendiente para futuras investigaciones el análisis de la posible abolladura elastoplástica de los soportes, que podría conducir a un fallo prematuro del sistema y a su fracaso. Ello podría poner en duda los espesores de $2 \mathrm{~mm}$ utilizados en algunas de las simulaciones numéricas aquí descritas.

\section{REFERENCIAS}

(1) Spieker, W. (1960). Versuche mit schutznetzen. Zeitschr. Berufsgenoss, 4, S4/7.

(2) Becker, K., Schmulling. (1973). Bericht zum Forschungsauftrag zur Ermittlung von Beurteilungs grundlagen für schutznetze Seilprüfstelle. Institut für Förderstechnik und Werkstoff Prüfung. Bochum.

(3) Lefevre, J. (1970). Considérations théoriques sur l'évaluation des effets dynamiques. Cahiers de notes documentaires, 671.

(4) Paureau, J. (1987). Filets carrés de securité en grandes nappes en polyamide 6 . Performances. Règles de pose. Institut National de Recherche et Sécurité. Vandoeuvre-lesNancy.

(5) Paureau, J., Parisot, E., Schuler, B. (1989). Filets en grandes nappes. Eficacité, performances, règles de pose. Cahiers de notes documentaires, 137 pp. 655-675.

(6) Saiz, J., García, R., Irles, R., Montava, I, Pey, J. (1994). Sobre las redes de seguridad. Salud y Trabajo, 104.

(7) Saiz, J., Irles, R., Arcenegui, G.A., Naharro, M. (1997). Las Redes de Seguridad en la Construcción. Universidad de Alicante y D.G. T. (Generalitat Valenciana), Alicante.

(8) Irles Más, R., González Sánchez, A., Segovia Eulogio, E., Maciá Mateu, A. (2002). Las redes verticales de seguridad en la construcción de edificios I. Informes de la Construcción, 53(477): 21-29. doi:10.3989/ic.2002.v53.i477.639.

(9) Segovia Eulogio, E., Irles Más, R., González Sánchez, A., Maciá Mateu, A.; Pomares Torres, J.C. (2007). Las redes verticales de seguridad en la construcción de edificios II. Informes de la Construcción, 59(505): 37-51. doi:10.3989/ic.2007.v59.i505.499.

(10) Lozano, C. (2005). Nueva normativa de sistemas de protección de borde y su aplicación práctica en las Obras. Revista del Colegio de Aparejadores y Arquitectos Técnicos de Alicante, 37, pp. 12-17.

(11) González, M. N., Cobo, A., Fuente, J. V., Bresó, S., Lozano, C. (2010). Comportamiento bajo cargas estáticas de sistemas provisionales de protección de borde realizados con elementos de acero. Informes de la Construcción, 63 (521): 57-67. doi:10.3989/ ic.09.070.

(12) Ordenanza General de Seguridad e Higiene en el Trabajo. B.O.E. n. ${ }^{\circ} 64$ y 65, 1971.

(13) Bellmunt Bellmunt, J.J. (1985). Notas técnicas de Prevención. NTP-24 INSHT, pp. 1-8.

(14) UNE 81.650.80. (1980). Redes de Seguridad, Características y Ensayos. AENOR.

(15) Ley 31/1995 por la que se aprueba la Ley de Prevención de riesgos laborales. Modificada por: (1) Ley 39/1999, (2) Real Decreto Legislativo 5/2000-Resolución de 16 de octubre de 2001, (3) Ley 54/2003, (4) RD 171/2004 y (5) RD 306/2007.

(16) UNE-EN 1263. (2002). Redes de Seguridad. Parte 1: Requisitos de seguridad, métodos de ensayo. AENOR.

(17) UNE-EN 1263. (2004). Redes de Seguridad. Parte 2: Requisitos de seguridad para la instalación de redes de seguridad. AENOR.

(18) UNE-EN 13374. (2004). Sistemas provisionales de protección de borde. Especificaciones del producto, método de ensayo. AENOR. 